Research Paper

\title{
Effectiveness of Cetuximab in Combination with Concurrent Chemoradiotherapy in Locoregionally Advanced Nasopharyngeal Carcinoma: A 1:2 Propensity Score-matched Analysis
}

\begin{abstract}
Li-Rong $\mathrm{Wu}^{*}$, Huan-feng Zhu*, Jianhua $\mathrm{Xu}^{*}$, Xue-song Jiang, Li Yin, Ning Jiang, Dan Zong, Fei-jiang Wang, Sheng-fu Huang, Xiu-hua Bian, Jian-feng Wu, Dan Song, Wen-jie Guo, Ju-Ying Liu ${ }^{\bowtie}, X_{i a} \mathrm{He}^{\bowtie}$

Department of Radiation Oncology, Nanjing Medical University Affiliated Cancer Hospital, Jiangsu Cancer Hospital, Jiangsu Institute of Cancer Research, 42\# Baiziting Road, Xuanwu District, Nanjing 210009, China

* These authors contributed equally to this work.

$\triangle$ Corresponding author: Ju-Ying Liu, M.D. juyingliu@21cn.com; Xia He, M.D. PhD. hexia206@yeah.net; Department of Radiation Oncology, Nanjing Medical University Affiliated Cancer Hospital, Jiangsu Cancer Hospital, Jiangsu Institute of Cancer Research, 42\# Baiziting Road, Xuanwu District, Nanjing 210009, China

(c) Ivyspring International Publisher. This is an open access article distributed under the terms of the Creative Commons Attribution (CC BY-NC) license (https://creativecommons.org/licenses/by-nc/4.0/). See http://ivyspring.com/terms for full terms and conditions.
\end{abstract}

Received: 2017.11.23; Accepted: 2018.01.19; Published: 2018.04.18

\begin{abstract}
Background: This study aimed to compare concurrent chemoradiotherapy (CCRT) plus cetuximab (C) with CCRT alone in locoregionally advanced nasopharyngeal carcinoma(NPC).

Methods: A total of 682 locoregionally advanced NPC patients who had undergone chemoradiotherapy with or without cetuximab were included. Propensity score-matching method was used to match patients. Progression-free survival (PFS), overall survival (OS), locoregional relapse-free survival (LRFS), and distant metastasis-free survival (DMFS) were compared between the two treatment arms.

Results: After matching, 225 patients were identified for the analysis. Compared to CCRT, CCRT plus $C$ was associated with significantly improved 3 -year PFS (83.7\% vs $71.9 \%, P=0.036)$, LRFS ( $98.6 \%$ vs $90.2 \%, P=0.034$ ) but not OS ( $91.4 \%$ vs $85.4 \%, P=0.117$ ). Among patients with T4 and/or N3 category, CCRT plus C significantly prolonged 3 -year PFS $(81.0 \%$ vs $61.4 \%, P=0.022)$ and increased 3-year OS (88.0\% vs $77.9 \%, P=0.086)$. No significant differences were observed between CCRT plus C and CCRT alone groups with regard to 3 -year PFS, OS, LRFS and DMFS rates in stage III patients. Acute oral and oropharyngeal mucositis during radiotherapy were more common in the CCRT plus $C$ than that in CCRT, but late toxicities were comparable.

Conclusions: This study reveals that patients with locoregionally advanced NPC could benefit from the addition of cetuximab to CCRT, and this therapeutic gain mainly originated from T4 and/or N3 subgroup although suffering more acute moderate to severe toxicities.
\end{abstract}

Key words:

\section{Introduction}

Nasopharyngeal carcinoma (NPC), a malignancy with an inimitable geographical distribution, is uncommon in Western countries [1], but is highly endemic in Southeast China [2]. More than 70\% of patients presented with locoregionally advanced disease at their initial diagnosis [3, 4], and prognosis is still unsatisfactory for these patients although radiotherapy techniques has been greatly reformed [1]. Concurrent chemoradiotherapy (CCRT) is the present standardized therapy for locoregionally 
advanced NPC [5-11]. However, there is increasing evidence showing that CCRT alone may be inadequate for absolute patients at higher risk, especially those with late clinical stage diseases who have a high potential for recurrence and metastasis [12]. Therefore, preferable therapeutic regimens are urgently demanded to further improve the therapeutic outcomes of patients with locoregionally advanced NPC.

Previous study revealed the epidermal growth factor receptor (EGFR) was highly expressed in $80 \%$ of locoregionally advanced NPC disease and correlated with poor survival [13]. Cetuximab, an anti-EGFR antibody, has been proven to improve survival outcomes of patients with locoregionally advanced head and neck squamous cell carcinoma (HNSCC) when combined treatment of cetuximab and radiotherapy (RT) was delivered [14]. It has been reported that the growth inhibitory effect of cisplatin can be improved by cetuximab in NPC cell lines [15]. Ma et al. have shown a Phase II clinical trial [16], which is the first study to have incorporated cetuximab into chemoradiotherapy for locoregionally advanced NPC. Concurrent treatment of cetuximab, weekly cisplatin, and intensity modulation radiated therapy (IMRT) is believed to be a feasible strategy. Our previous work had observed that for locoregionally advanced NPC patients concurrent administration of cetuximab, chemotherapy, and IMRT was effective and tolerated [17]. Until now, no randomized controlled clinical trial has been proved the effectiveness of that combined treatment. Accordingly, it is still undecided whether patients of locoregionally advanced NPC can benefit from the combination of CCRT plus C.

Therefore, we conducted this retrospective investigation to compare the efficacies and toxicities of CCRT plus $\mathrm{C}$ with CCRT alone in locoregionally advanced NPC patients using the propensity score-matching (PSM) method, which is usually conceptualized to be imitated randomized trials.

\section{Materials and method}

\section{Patients}

There were 681 consecutive patients with newly diagnosed stage III-IVB NPC between January 2010 and December 2014 were included in this retrospective study. All disease was non-keratinized squamous cell carcinoma. Among them, 75 underwent CCRT with cetuximab and 606 received CCRT alone. All patients had entire assessments before treatment, including history, physical examinations, hematology and biochemistry profiles, abdominal sonography, electrocardiogram (ECG), chest radiography, magnetic resonance imaging (MRI) of the neck and nasopharynx, fiber optic nasopharyngoscopy with biopsy, and technetium99m-methylene diphosphonate (Tc-99-MDP) wholebody bone scans. Based on these examinations, patients were restaged according to the seventh edition of the American Joint Committee on Cancer/International Union against Cancer (AJCC/UICC) staging system. This study was approved by the the Research Ethics Committee of Jiangsu Cancer Hospital. Written informed consent was obtained from all the patients before treatment. All methods of this study were performed in accordance with the relevant guidelines and regulations.

\section{Treatments}

The nasopharyngeal and neck tumor of all patients were treated by intensity modulation radiated therapy (IMRT), and 9-11 radiation fields were used for the IMRT. The prescribed doses were 66-75 Gy at 2.10-2.25 Gy/fraction to the planning target volume (PTV) of the primary gross tumor volume, 64-72 Gy per 28-33 fractions to the PTV of the involved lymph nodes volume, 60-62 Gy per 28-31 fractions to the PTV of the high-risk clinical target volume, and 50-52 Gy per 25-30 fractions to the PTV of the low-risk clinical target volume. Salvage treatments (chemotherapy, surgery or brachytherapy) were supplied in recorded recurrences or residual diseases whenever possible. Radiotherapy boost after primary external radiotherapy was received in $11 \%$ of these patients.

During the period of this study, official guidelines suggested CCRT for stage III-IVb with or without induction/adjuvant chemotherapy, and it was specified by the seventh editions of the AJCC/UICC TNM staging system of NPC. Overall, 75 patients in the CCRT plus $C$ group received two cycles of concurrent chemotherapy consisting of paclitaxel and nedaplatin (TP). Among 606 patients without cetuximab, 9/606 (1.5\%) were treated with RT only and 597/606 (98.5\%) received RT and chemotherapy (concurrent alone). In our institution, concurrent chemotherapy regimens included docetaxel $\left(70 \mathrm{mg} / \mathrm{m}^{2} \mathrm{~d} 1\right)$ with cisplatin $\left(60 \mathrm{mg} / \mathrm{m}^{2}\right.$ $\mathrm{d} 1-3)$ (TP), or 3-weekly cisplatin $\left(80 \mathrm{mg} / \mathrm{m}^{2}\right.$ $\mathrm{d} 1-3)$ /nedaplatin $\left(80 \mathrm{mg} / \mathrm{m}^{2} \mathrm{~d} 2-4\right)$.

The therapy regimen of CCRT with cetuximab was determined by our previous study [17]. From the first day of radiotherapy, Cetuximab was used at a $250 \mathrm{mg} / \mathrm{m} 2$ on a weekly basis $(400 \mathrm{mg} / \mathrm{m} 2$ initial dose) for 7 times. Cetuximab was given by intravenous infusion over $120 \mathrm{~min}$ in the first week and over 60 min in the successive radiotherapy weeks. 


\section{Toxicity and Follow-up}

Acute and late toxicities were graded in term of the National Cancer Institute Common Toxicity Criteria for Adverse Events version 3.0 and the Radiation Therapy Oncology Group (RTOG) Late Radiation Morbidity Scoring Criteria, respectively [18].

The follow-up was counted from the day of first treatment to the day of last examination or death. In Jiangsu Cancer Hospital a standard follow-up contains complaints query, physical examinations, indirect or direct nasopharyngoscopy, abdominal sonography and chest $\mathrm{x}$-ray. It was demanded with head and neck MRI every three months in the first three years, then MRI every six months after three years. Except for recurrence at the skull base, which was confirmed by bone erosion on MRI, all local recurrences were identified by soft-tissue swelling in MRI of the nasopharynx fiber optic endoscopy and diagnosed by biopsy. We defined distant metastases using symptoms, physical examinations, and bone scan, CT scan, chest X-ray, abdominal sonography, and MRI. We diagnosed the regional recurrences using biopsy, neck MRI and clinical examination.

\section{Statistical analysis}

PSM method [19] was adopted to match patients between these two groups at a 1:2 ratio using the following factors: gender, age, histology, body mass index (BMI), titers of immunoglobulin A against early antigen (EBV-EA-lgA) and immunoglobulin A against viral capsid antigen (EBV-VCA-lgA) of the Epstein-Barr (EB) virus, chemotherapy regimen, T-stage, and N-stage. Covariate balances between the 2 groups were checked by the chi-squared test (categorical variable), t-test (continuous variable), or Fisher's exact test as appropriate.

Progression-free survival (PFS), overall survival (OS), locoregional relapse-free survival (LRFS) and distant metastasis-free survival (DMFS) were analyzed using the Kaplan-Meier method and compared with the log-rank test. PFS was calculated from the first day of treatment to the day of disease progression or death from any cause; OS, to death or the last examination; LRFS, to the day of locoregional failure; and DMFS, to the day of metastasis. Backward exclusion of negligible explicative variables were implemented to analyze for independent significance using Cox proportional hazards model to implement multivariate analyses.

The statistical analyses were executed by the SPSS 23.0 (Chicago, IL, USA) and R software version 2.15.3 (R Foundation for Statistical Computing, Vienna, Austria; www.r-project.org). Two-sided $P$ values $<0.05$ was considered significant.

\section{Results}

\section{Patients selection}

There were 681 patients included in this study. The median duration of follow-up was 45.3 months. Statistically marked diversities were found regarding age $(P<0.001)$, EBV-EA-lgA $(P=0.034)$, T-stage $(P=$ $0.004)$, and N-stage $(P=0.033)$ when stratifying patients based on initial treatment (CCRT + C vs CCRT). After matching, 150 (66.7\%) patients were treated with CCRT and 75 (33.3\%) with CCRT + C were selected, and balanced features were also studied (containing the mean age, sex, BMI, histology, EBV-EA-lgA and EBV-VCA-lgA titers, T-stage, $\mathrm{N}$-stage, clinical stage). Table 1 shows the the baseline characteristics of patients between these two groups.

Table 1. Baseline characteristics of nasopharyngeal carcinoma patients who underwent chemoradiotherapy with or without cetuximab

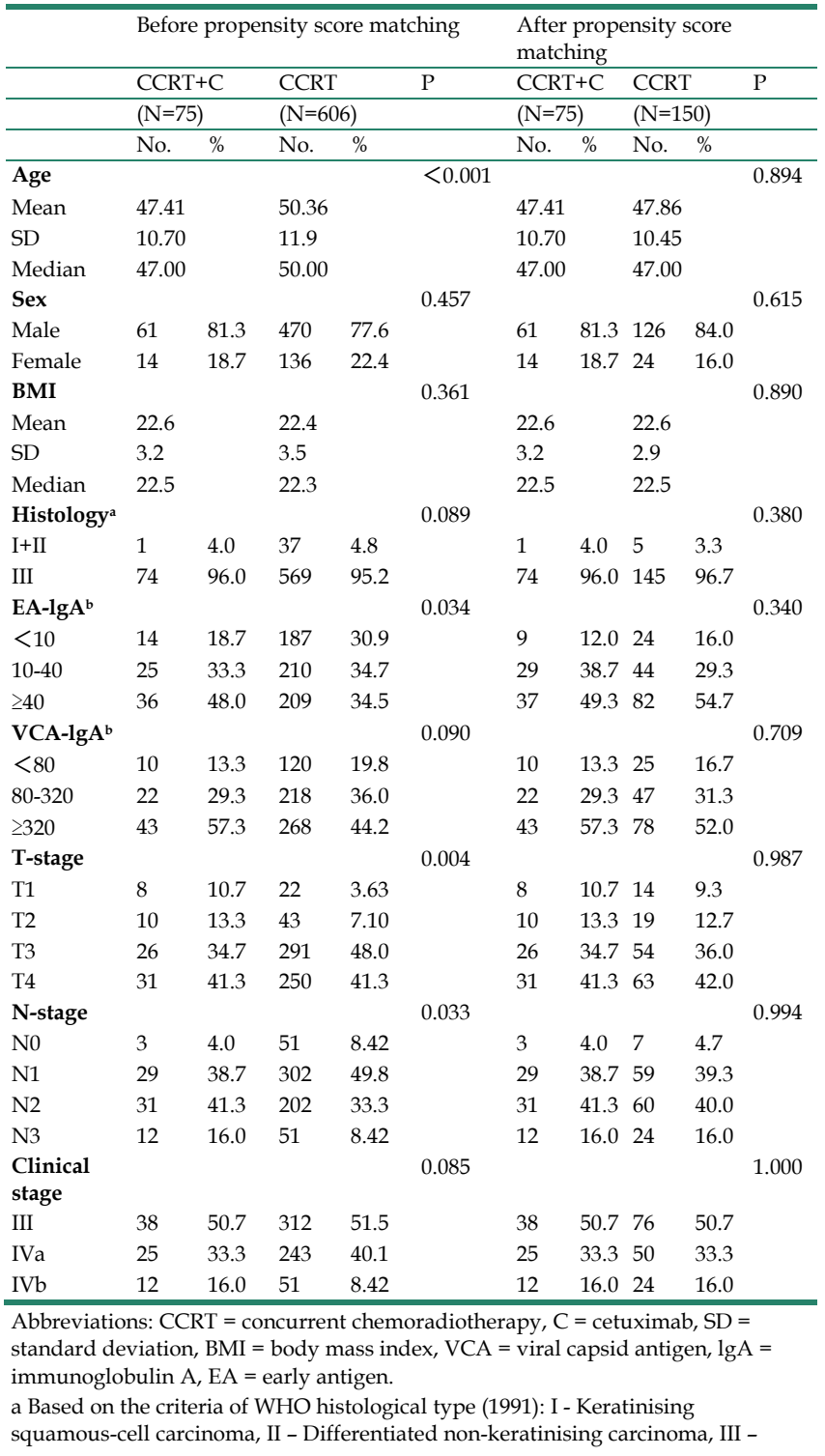


Undifferentiated non- keratinising carcinoma.

${ }^{\mathrm{b}}$ In accordance with the criteria adopted in previous studies.

c Used Fisher's exact test.

\section{Survival outcomes}

In the original unmatched cohort of 681 patients, the median follow-up time was 43.9 months (5.4-78.6 months) for the CCRT arm and 41.0 months (6.0-71.2 months) for the CCRT $+\mathrm{C}$ arm, respectively. The 3 -year PFS $(83.9 \%$ vs $76.6 \%, P=0.153)$, OS $(86.4 \%$ vs $91.4 \%, P=0.284)$, LRFS $(93.2 \%$ vs $98.6 \%, P=0.784)$, and DMFS $(84.3 \%$ vs $83.9 \%, P=0.918)$ rates were comparable between CCRT alone and CCRT plus C groups (Figure S1 in the Supplementary Appendix, available at Journal of Cancer).

Table 2. Summary of important prognostic factors in the multivariate analysis in the propensity-matched cohort. ${ }^{a}$

\begin{tabular}{|c|c|c|}
\hline & Hazard ratio $(95 \% \mathrm{CI})$ & $P$ \\
\hline \multicolumn{3}{|l|}{ Progressive-free survival } \\
\hline $\mathrm{CCRT}+\mathrm{C}$ versus CCRT & $1.26(1.16-2.79)$ & 0.015 \\
\hline Sex & $0.51(0.25-1.05)$ & 0.069 \\
\hline Age (continuous) & $0.80(0.46-1.38)$ & 0.071 \\
\hline BMI (continuous) & $1.04(0.79-2.87)$ & 0.130 \\
\hline Clinical stage & $1.40(1.23-1.90)$ & $<0.001$ \\
\hline T-stage & $1.21(1.17-1.86)$ & 0.023 \\
\hline $\mathrm{N}$-stage & $1.46(1.53-2.26)$ & 0.015 \\
\hline \multicolumn{3}{|l|}{ Overall survival } \\
\hline CCRT $+C$ versus CCRT & $1.14(0.83-3.87)$ & 0.109 \\
\hline Sex & $0.40(0.15-1.05)$ & 0.162 \\
\hline Age (continuous) & $0.65(0.32-1.30)$ & 0.068 \\
\hline BMI (continuous) & $0.74(0.49-1.87)$ & 0.072 \\
\hline Clinical stage & $1.32(1.16-2.45)$ & $<0.001$ \\
\hline T-stage & $1.76(1.29-2.37)$ & 0.007 \\
\hline N-stage & $2.04(1.53-2.59)$ & 0.001 \\
\hline \multicolumn{3}{|c|}{ locoregional relapse-free survival } \\
\hline $\mathrm{CCRT}+\mathrm{C}$ versus $\mathrm{CCRT}$ & $1.19(0.53-1.70)$ & 0.047 \\
\hline Sex & $0.62(0.21-1.87)$ & 0.242 \\
\hline Age (continuous) & $0.58(0.33-1.15)$ & 0.264 \\
\hline BMI (continuous) & $0.82(0.41-1.38)$ & 0.108 \\
\hline Clinical stage & $1.53(0.91-2.43)$ & 0.075 \\
\hline T-stage & $1.97(1.32-2.81)$ & 0.013 \\
\hline $\mathrm{N}$-stage & $0.78(0.21-1.36)$ & 0.569 \\
\hline \multicolumn{3}{|c|}{ Distant metastasis-free survival } \\
\hline $\mathrm{CCRT}+\mathrm{C}$ versus CCRT & $0.76(0.77-1.98)$ & 0.242 \\
\hline Sex & $0.96(0.52-1.77)$ & 0.694 \\
\hline Age (continuous) & $0.71(0.47-1.30)$ & 0.145 \\
\hline BMI (continuous) & $0.43(0.37-1.04)$ & 0.127 \\
\hline Clinical stage & $1.22(0.62-1.16)$ & 0.001 \\
\hline T-stage & $0.53(0.30-1.45)$ & 0.116 \\
\hline $\mathrm{N}$-stage & $2.24(1.14-2.76)$ & $<0.001$ \\
\hline
\end{tabular}

In the propensity-matched regiment of 225 patients, 41.0 months (6.0-71.2 months) for the CCRT plus $C$ arm and 43.6 months (5.6-75.3 months) for the CCRT arm were shown in the median follow-up time. In the univariate analysis, the 3-year PFS rates $83.7 \%$ vs $72.0 \%, P=0.036)$ and 3 -year LRFS rates $(98.6 \%$ vs $90.2 \%, P=0.034)$ were significantly higher for patients with the CCRT plus $C$ arm than with CCRT alone.
Furthermore, a marginal trend of increasing risk of 3 -year DMFS rates $(83.9 \%$ vs $78.4 \%, P=0.301)$ and 3 -year OS rates $(91.2 \%$ vs $85.8 \%, P=0.123)$ was found (Figure 1). The results of the multivariate analysis indicated that CCRT plus C treatment was a significant and independent protective predictor for 3 -year PFS $(\mathrm{HR}=1.26,95 \%$ CI 1.164-2.789. $P=0.015)$ and LRFS rates $(\mathrm{HR}=1.19,95 \%$ CI 0.525-1.703. $P=$ 0.047) (TABLE 2).

\section{Subgroup analysis}

When focusing on patients with stage T4 and/or $\mathrm{N} 3$ in the subgroup, the CCRT plus $\mathrm{C}$ arm achieved significantly prolonged 3 -year PFS $(79.9 \%$ vs $62.6 \%, P$ $=0.022)$ and a marginally increased OS $(88.0 \%$ vs $77.9 \%, P=0.086$ ) (Figure 2) compared with that of CCRT alone arm. Additionally, the 3-year LRFS $(97.0 \%$ vs $90.9 \%, P=0.246)$ and DMFS $(79.9 \%$ vs $67.8 \%, P=0.161)$ were marginally enhanced in patients with CCRT plus $C$ than in those with CCRT (Figure 2). The outcome of the multivariate analysis proved that cetuximab also reduced the risk of disease progression $(\mathrm{HR}=2.34 \mathrm{CI} 1.08-5.06, P=0.031)$ (Table $3)$.

When concentrating on stage III patients, there were no considerable statistically diversities found in 3-year PFS, OS, LRFS, and DMFS rates between patients with and without cetuximab treatment (Figure 3, Table 3).

\section{Treatment toxicity}

No treatment-related deaths occurred in this study. Acute toxic effects induced by the drugs of this study were mostly controllable and reversible. The most usually observed grade 3-4 hematological toxic effects were lymphopenia and neutropenia which were not normally accompanied by any vital infections. Chemotherapy induced pathognomonic toxic effects were rare. However, in the treatment with CCRT plus $C$, acneiform rash was the most usually observed grade 3-4 cetuximab-related toxicity. In $16(21.3 \%)$ CCRT plus C patients, Dermatitis (radiation induced) was recorded as grade 3-4, which was more often than with CCRT alone $2(1.3 \%, \mathrm{P}<$ 0.001). A few patients had creatinine elevation or alanine transferase elevation in these two arms (Table $4)$.

Late toxicities occurrence at the last follow-up were summarized in Table 4 . The most usually observed late toxic effects was Xerostomia. No cases of radiation-induced neuropathy were observed in this study. No significant difference was shown in the late toxicities of xerostomia, endocrine, neuropathy, sensorineural hearing loss, osteonecrosis, or radiation encephalopathy between the two treatment arms. 
A

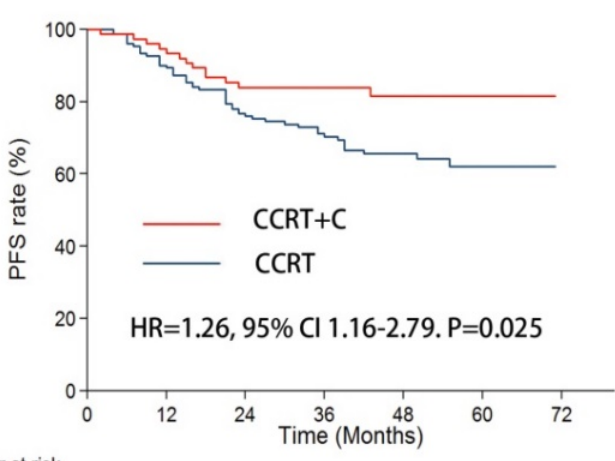

Number at risk

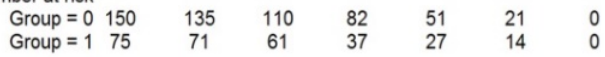

C
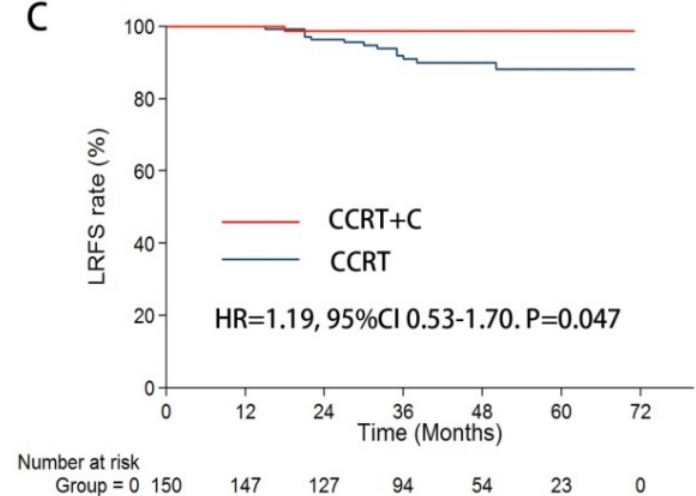

B

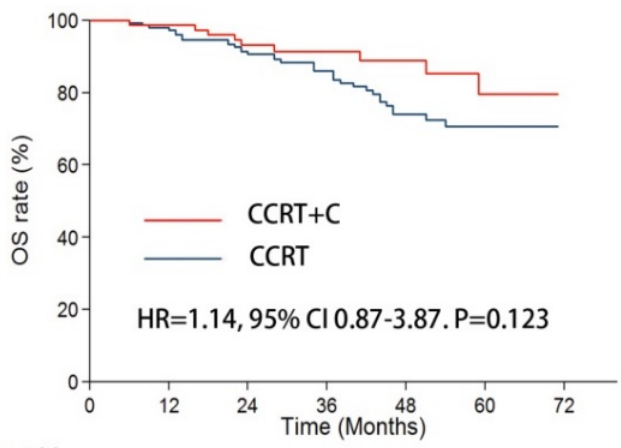

Number at risk

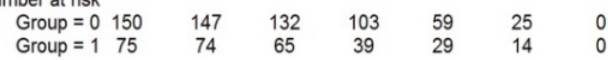

D
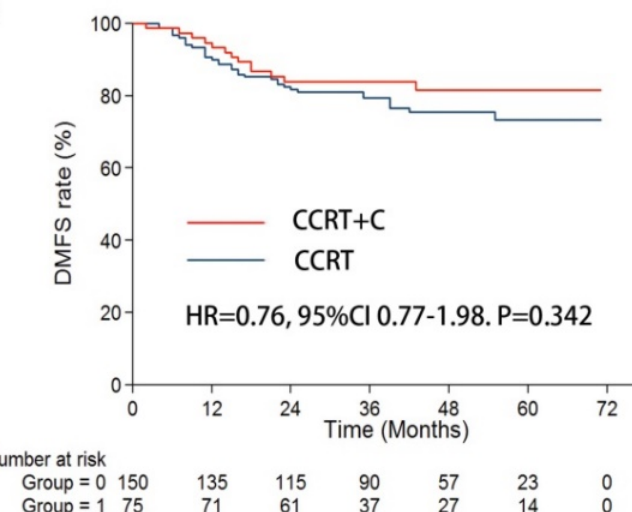

Figure 1. Kaplan-Meier survival curves for the CCRT + C arm and CCRT arm in the propensity-matched cohort of 225 patients. A. PFS; B. OS; C. LRFS; D. DMFS.

A

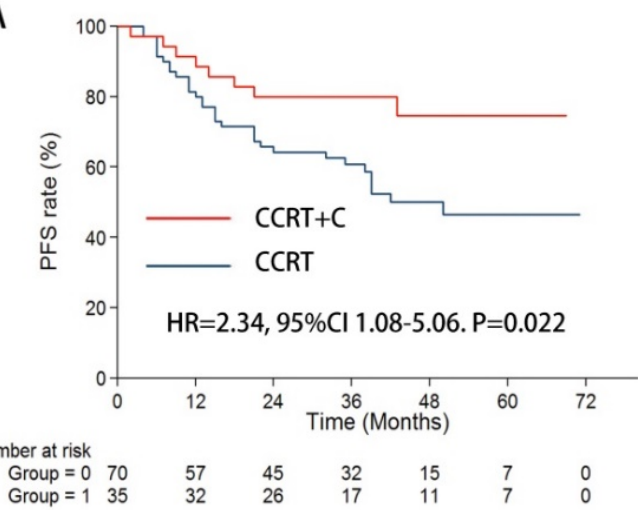

\section{C}

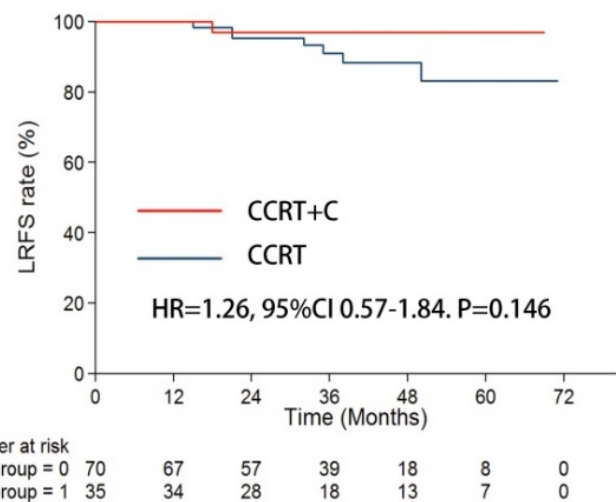

B

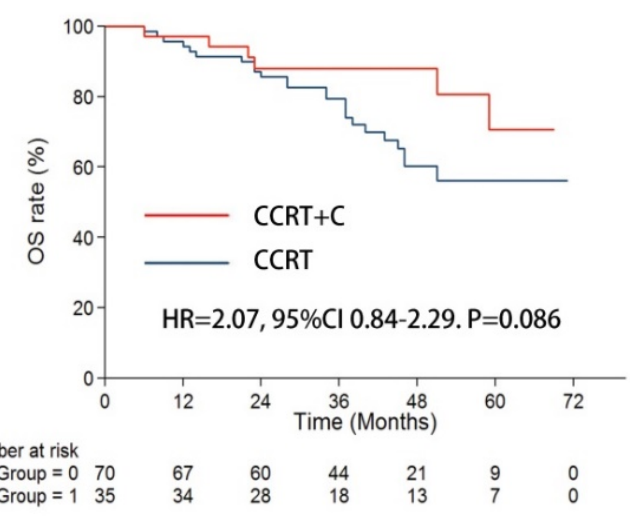

D

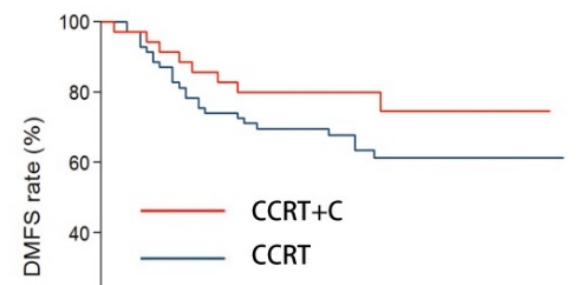

$\mathrm{HR}=0.69,95 \% \mathrm{Cl} 0.38-2.14 . \mathrm{P}=0.161$

Number at risk

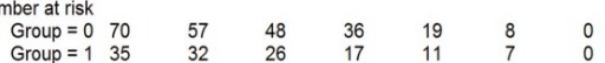

Figure 2. In the subgroup of stage T4 and/or N3, Kaplan-Meier survival curves for the CCRT + C arm and CCRT arm. A. PFS; B. OS; C. LRFS; D. DMFS. 
A

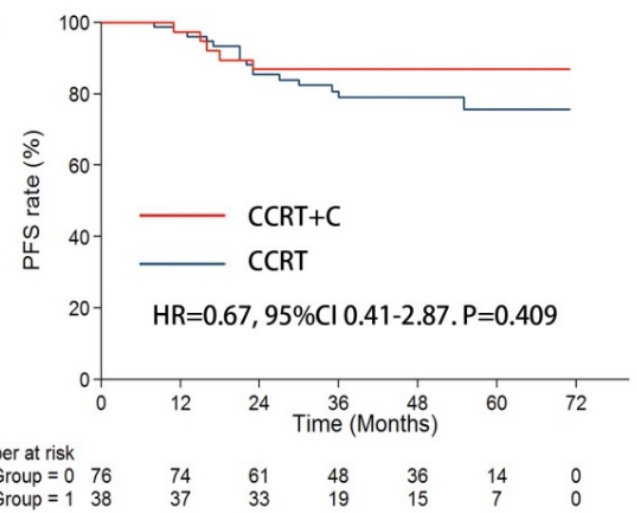

C

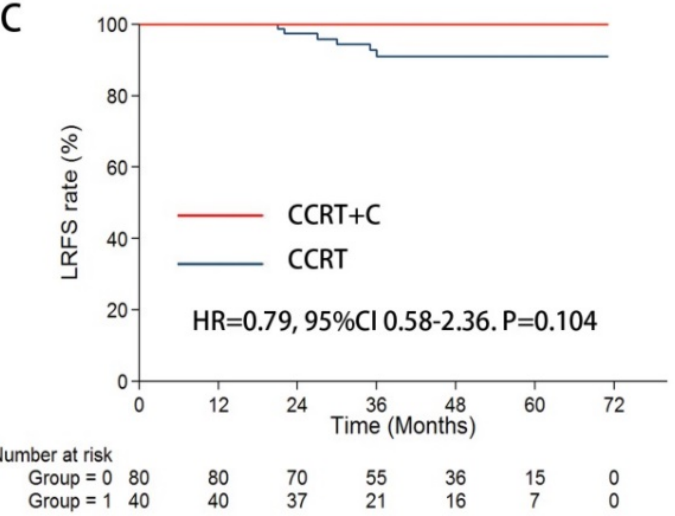

B

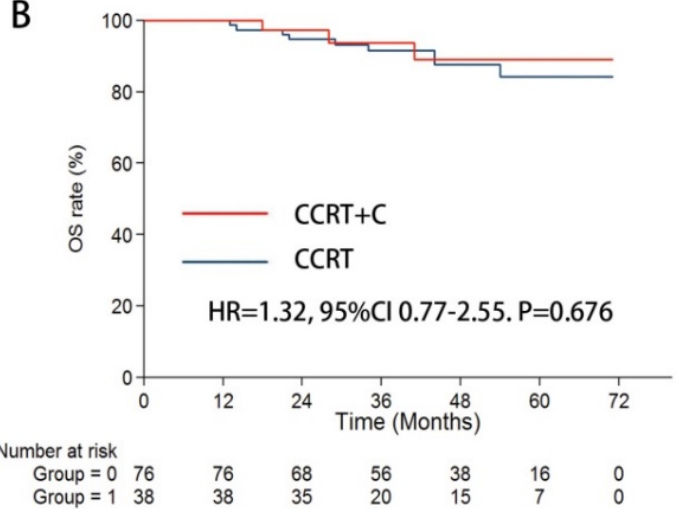

D

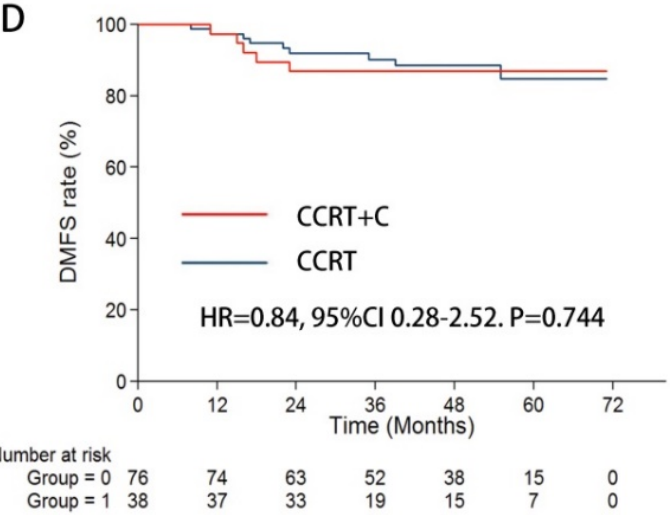

Figure 3. In the subgroup of stage III, Kaplan-Meier survival curves for the CCRT + C arm and CCRT arm. A. PFS; B. OS; C. LRFS; D. DMFS.

Table 3. Subgroup analysis by prognostic factors in the multivariate analysis in the propensity-matched cohort. ${ }^{\mathrm{a}}$

\begin{tabular}{|c|c|c|c|c|c|c|c|c|c|}
\hline & \multirow{2}{*}{$\begin{array}{l}\mathrm{N} \\
(\mathrm{CCRT}+\mathrm{C} \\
\text { vs CCRT) }\end{array}$} & \multicolumn{2}{|l|}{$\begin{array}{l}\text { Progressive-free } \\
\text { survival }\end{array}$} & \multicolumn{2}{|l|}{ Overall survival } & \multicolumn{2}{|l|}{$\begin{array}{l}\text { Locoregional } \\
\text { relapse-free survival }\end{array}$} & \multicolumn{2}{|l|}{$\begin{array}{l}\text { Distant metastasis- } \\
\text { free survival }\end{array}$} \\
\hline & & Hazard ratio $(95 \% \mathrm{CI})$ & $P$ & Hazard ratio $(95 \% \mathrm{CI})$ & $P$ & Hazard ratio $(95 \% \mathrm{CI})$ & $P$ & Hazard ratio $(95 \% \mathrm{CI})$ & $P$ \\
\hline \multicolumn{10}{|l|}{ Age } \\
\hline$<45$ ys & 28 vs 54 & $1.34(0.58-1.46)$ & 0.143 & $0.58(0.63-1.93)$ & 0.247 & $1.18(0.32-1.73)$ & 0.165 & $1.24(0.61-1.47)$ & 0.420 \\
\hline$\geq 45 y s$ & 47 vs 96 & $1.79(0.89-2.08)$ & 0.058 & $0.81(0.73-1.57)$ & 0.233 & $1.31(0.61-1.81)$ & 0.087 & $1.07(0.63-2.19)$ & 0.483 \\
\hline \multicolumn{10}{|l|}{ Sex } \\
\hline Male & 61 vs 121 & $1.39(0.32-2.11)$ & 0.047 & $1.06(0.76-1.48)$ & 0.215 & $1.43(0.42-1.29)$ & 0.046 & $1.22(0.63-2.10)$ & 0.554 \\
\hline Female & 14 vs 29 & $1.15(0.48-1.67)$ & 0.356 & $0.58(0.21-1.64)$ & 0.670 & $0.50(0.26-0.95)$ & 0.150 & $1.08(0.61-1.89)$ & 0.781 \\
\hline \multicolumn{10}{|l|}{ Stage } \\
\hline III & 38 vs 76 & $0.67(0.414-2.87)$ & 0.409 & $1.32(0.77-2.25)$ & 0.676 & $0.67(0.41-2.87)$ & 0.105 & $0.84(0.28-2.52)$ & 0.756 \\
\hline T4 and/or N3 (IVa+IVb) & 37 vs 74 & $2.34(1.08-5.06)$ & 0.031 & $2.07(0.84-2.29)$ & 0.110 & $1.26(0.57-1.84)$ & 0.049 & $0.69(0.38-2.14)$ & 0.181 \\
\hline $\mathrm{N} 3$ (IVb) & 12 vs 24 & $1.85(0.39-2.42)$ & 0.487 & $1.86(0.61-1.98)$ & 0.045 & $1.36(0.53-4.20)$ & 0.489 & $1.53(0.21-2.64)$ & 0.292 \\
\hline
\end{tabular}

Abbreviations: $\mathrm{CCRT}=$ concurrent chemoradiotherapy, $\mathrm{C}=$ cetuximab, $\mathrm{CI}=$ confidence interval.

a Adjusted for clinical stage with Enter method, age and sex with Backward LR method.

\section{Discussion}

In the present study, we demonstrated that CCRT plus cetuximab was associated with significantly improved 3-year PFS (83.7\% vs $71.9 \%)$ and LRFS rates $(98.6 \%$ vs $90.2 \%)$ compared with CCRT alone in locoregionally advanced NPC, and this benefit mainly originated from patients with bulky lesions and/or extensive nodal diseases (T4 and/or N3). Presently, the propensity score-matching analysis, together with multivariate analysis, supplies the most objectively comparable results from matched patients with the treatment of CCRT with or without cetuximab to evaluate the efficacy of cetuximab.

EGFR is overexpressed and upregulated in more than $80 \%$ HNSCC tumors [20]. Cetuximab, an anti-EGFR monoclonal antibody, intensifies the sensitivity to chemotherapy and/or radiotherapy and the stimulation of antibody-dependent cellular cytotoxicity (ADCC) in HNSCC [21, 22]. In preclinical studies of HNSCC, several antitumor effects of cetuximab have been proved, those contain the inhibition of cell growth/survival and metastasis, angiogenesis [23]. Moreover, previous studies had 
been presented that cetuximab is safe and effective in locally advanced HNSCC patients. The advantage of combined strategies of cetuximab with RT regarding locoregional control (3 years, $47 \%$ vs $34 \%$ ) and OS (5 years, $46 \%$ vs $36 \%$ ) in locally advanced HNSCC was shown in Bonner's study [14]. When concomitantly delivered with RT in locally advanced HNSCC [14], it confirmed that cetuximab could significantly improve survival. Additionally, for patients with locoregionally advanced HNSCC, a randomized trial demonstrated that cetuximab plus radiotherapy considerably improved 5-year OS. It provided supplementary support for the current addition of cetuximab in the NCCN Guidelines as a recognized standard systemic treatment for locally advanced HNSCC patients [24]. Notwithstanding, for stage III-IV HNSCC patients in concurrent radiochemotherapy with or without cetuximab, phase III trial (RTOG 0522) [25], cetuximab could significantly improve the 2-year OS and PFS. Recently, researchers have begun to search for more combinations of therapies and subgroups that are sensitive to cetuximab [26].

Table 4. Incidence of major acute and late toxicities in the propensity matched cohort. ${ }^{\mathrm{a}}$

\begin{tabular}{|c|c|c|c|c|c|}
\hline \multirow[t]{2}{*}{ Toxicity } & \multicolumn{2}{|c|}{$\begin{array}{l}\text { CCRT+C }(\mathrm{n}=75) \\
\text { No. of patients }(\%)\end{array}$} & \multicolumn{2}{|c|}{$\begin{array}{l}\text { CCRT }(\mathrm{n}=150) \\
\text { No. of patients }(\%)\end{array}$} & \multirow[t]{2}{*}{$P$ value } \\
\hline & Grade0-2 & Grade3-4 & Grade0-2 & Grade3-4 & \\
\hline \multicolumn{6}{|l|}{ Acute toxicities } \\
\hline \multicolumn{6}{|l|}{ Hematologic } \\
\hline Neutropenia & $37(49.3)$ & $38(50.7)$ & $83(55.3)$ & $67(44.7)$ & 0.400 \\
\hline Leucopenia & 35 (46.7) & $40(53.3)$ & $82(54.7)$ & $68(45.3)$ & 0.262 \\
\hline Thrombocytopenia & 73 (97.3) & $2(2.7)$ & 149 (99.3) & $1(0.7)$ & 0.258 \\
\hline Anemia & $74(98.7)$ & $1(1.3)$ & $150(100.0)$ & $0(0.0)$ & 0.333 \\
\hline \multicolumn{6}{|l|}{ Non-hematologic } \\
\hline Acneiform rash & $64(85.3)$ & $11(14.7)$ & $150(100.0)$ & $0(0.0)$ & $<0.001$ \\
\hline $\begin{array}{l}\text { Dermatitis (radiation } \\
\text { induced) }\end{array}$ & 59 (78.7) & $16(21.3)$ & 148 (98.7) & $2(1.3)$ & $<0.001$ \\
\hline Oral mucositis & $25(33.3)$ & $50(66.7)$ & $135(90.0)$ & $15(10.0)$ & $<0.001$ \\
\hline Vomiting & $67(89.3)$ & $8(10.7)$ & $150(100.0)$ & $0(0.0)$ & $<0.001$ \\
\hline Weight loss & $60(80.0)$ & $15(20.0)$ & $128(85.3)$ & $22(14.7)$ & 0.342 \\
\hline Creatinine elevation & $75(100.0)$ & $0(0.0)$ & $150(100.0)$ & $0(0.0)$ & 1.000 \\
\hline $\begin{array}{l}\text { Alanine transferase } \\
\text { elevation }\end{array}$ & $75(100.0)$ & $0(0.0)$ & 149 (99.3) & $1(0.7)$ & 1.000 \\
\hline \multicolumn{6}{|l|}{ Late toxicities } \\
\hline Xerostomia & $65(86.7)$ & $10(13.3)$ & $128(85.3)$ & $22(14.7)$ & 1.000 \\
\hline Endocrine & 74 (98.7) & $1(1.3)$ & $147(98.0)$ & $3(2.0)$ & 1.000 \\
\hline Neuropathy & $75(100.0)$ & $0(0.0)$ & $150(100.0)$ & $0(0.0)$ & 1.000 \\
\hline $\begin{array}{l}\text { Sensorineural hearing } \\
\text { loss }\end{array}$ & $75(100.0)$ & $0(0.0)$ & 148 (98.7) & $2(1.3)$ & 0.554 \\
\hline Osteonecrosis & $75(100.0)$ & $0(0.0)$ & 149 (99.3) & $1(0.7)$ & 1.000 \\
\hline $\begin{array}{l}\text { Radiation } \\
\text { encephalopathy }\end{array}$ & 73 (97.3) & $2(2.7)$ & 145 (96.7) & $5(3.3)$ & 1.000 \\
\hline
\end{tabular}

Abbreviations: $\mathrm{CCRT}=$ concurrent chemoradiotherapy, $\mathrm{C}=$ cetuximab.

a Used Fisher's exact test.

Actually, it is confirmed that radiotherapy or chemotherapy combined with cetuximab has a higher tumor control rate and survival rate; this therapy strategy in HNSCC indicate the direction of NPC. However, it has to be analyzed prudently due to two types of tumors' various biological behaviors. A preliminary Phase II clinical trial outcome was recorded by Ma et al [16], which is the first study to have incorporated cetuximab into chemoradiotherapy for locoregionally advanced NPC. The combination therapy led to a 2-year PFS of $86.5 \%$, which was more effective than historic files according to the therapy without cetuximab (A 2-year PFS rate of $76 \%$ was reported in the same center). In this retrospective study, in order to decrease potential biases as much as possible, we used propensity score matching. In the propensity-matched cohort, the 3-year PFS rate $(83.7 \%$ vs $72.0 \%, P=0.036)$ and 3 -year LRFS rate $(98.6 \%$ vs $90.2 \%, P=0.034)$ of the patients with CCRT plus $C$ were considerably better compare with those with CCRT alone. Furthermore, our data showed that 3 -year DMFS rate $(83.9 \%$ vs $78.4 \%, P=0.301)$ and 3 -year OS rates $(91.2 \%$ vs $85.8 \%, P=0.123)$ were marginally improved, although no significant difference between the 2 treatments was observed with regard to the rates of DMFS and OS. However, from the addition of cetuximab to the CCRT in locoregionally advanced NPC, patients with T4 and/or N3 could gain greatly improve. We found that the OS was enhanced by above $10 \%$ in the subgroup of T4 and/or N3 stage with the regimen of CCRT plus C, although there were statistically insignificant differences. Furthermore, considerably lower risk of tumor progression and marginal progress in LRFS and DMFS were observed. Recently, Zhang et al.[27] reported survival rates in locoregionally advanced NPC rose by helical tomotherapy plus cetuximab, then introduced TP adjuvant chemotherapy. Moreover, cetuximab accompanied with chemoradiotherapy was well tolerated and effective in the treatment of metastatic nasopharyngeal carcinoma or recurrent [28]. Nevertheless, $\mathrm{Xu}$ et al.[29] showed that cetuximab radiotherapy did not enhance the 3-year OS compared with cisplatin-chemoradiotherapy in locoregionally advanced NPC. Wu et al.[30] evaluated cetuximab-radiotherapy was not inferior to traditional $\mathrm{CR}$ in a retrospective study,. These results are markedly different from ours, since these 2 studies enrolled mostly stage II/III patients. In our study, we observed that there were no statistically significant differences in 3-year PFS, OS, LRFS, and DMFS rates between the 2 groups, and patients with stage III were less likely to benefit from CCRT plus C. To the best of our knowledge, this is the first research reporting that a combination of cetuximab and CCRT can significantly increase PFS and OS in patients with stage T4 and/or N3 but rarely benefits stage III NPC patients.

We observed that oral and oropharyngeal mucositis were the most common acute toxic effects. 
Hematologic toxicity was not different between the two groups. No treatment-related deaths occurred. Moreover, our previous study reported more acute moderate to severe toxicities and dermal toxicities and oral mucosal responses in particular. This adverse reaction was specific to cetuximab and more toxic in combination with radiochemotherapy. Nevertheless, cetuximab and CCRT of locoregionally advanced NPC is effective and tolerant [17]. When contrast the concurrent RT plus cisplatin (either IMRT or 2D-RT), Ma et al. showed a comparatively high percentage of RT-related mucosal toxic effects and acute skin [16]. Another study presented that concurrent treatment of RT, cisplatin, and cetuximab, was a feasible strategy against locoregionally advanced NPC, which also showed the mucosal and skin toxic effects were controllable, improvable and invertible[31]. It has even been found that the head and neck carcinoma patient who had grade 2-4 cetuximab rash was normally observed higher survival rate [32]. Additionally, during this study, the recorded late toxic effects were normally grade 1-2 in severity; no significant difference was observed in the late toxicities. They were similar as the recorded respective rate in the study by Zeng et al [33].

By propensity score matching and multivariate analysis, with the largest sample size analysis of the CCRT plus $C$ effect in locoregionally advanced NPC, it demonstrates main advantage of this study. The restriction of correlative selection bias and divergent confounders from retrospective assessment of observational data has been exceedingly improved by this method. In spite of the statistics were collected from one single institution, they supplied beneficial advices on the CCRT plus C impact prior to a concluding report of any Phase III randomized controlled trial.

Our study has several limitations. The innate limitation of this single institutional retrospective study has been recognized. Due to in many circumstances, statistics on the EB - DNA copy number could not be located and recorded, they were replaced by EBV-EA-Ig A and EBV-VCA-Ig A. Therefore, in this retrospective study, it was difficult to acquire extensive toxicity statistics. Furthermore, a chosen group of malignant cases statistics is presented in this dissertation since there was no clinical trial applied toward these patients. Finally, it is necessary to interpret our results prudentially due to the relatively short follow-up time.

\section{Conclusion}

This propensity-matched study reveals that patients with $\mathrm{T} 4$ and/or N3 stage could benefit from the combination of cetuximab with the current chemoradiotherapy in locoregionally advanced NPC, although with more acute moderate to severe toxicities. However, this strategy remains to be validated in a prospective randomized controlled study.

\section{Abbreviations}

CCRT: concurrent chemoradiotherapy; C: cetuximab; NPC: nasopharyngeal carcinoma; PFS: progression-free survival; OS: overall survival; LRFS: locoregional relapse-free survival; DMFS: distant metastasis-free survival; EGFR: epidermal growth factor receptor; HNSCC: head and neck squamous cell carcinoma; RT: radiotherapy; IMRT: intensity modulation radiated therapy; PSM: propensity score-matching; ECG: electrocardiogram (ECG); MRI: magnetic resonance imaging; Tc-99-MDP: technetium-99m-methylene diphosphonate; AJCC/UICC: American Joint Committee on Cancer/International Union against Cancer; PTV: planning target volume; RTOG: Radiation Therapy Oncology Group; CT: Computed Tomography; BMI: body mass index; EBV-EA-lgA: immunoglobulin A against early antigen; EBV-VCA-lgA: immunoglobulin A against viral capsid antigen; EB: Epstein-Barr; SPSS: Statistical Product and Service Solutions; NCCN: National Comprehensive Cancer Network; SD: standard deviation, VCA: viral capsid antigen; $\lg \mathrm{A}$ : immunoglobulin A; EA: early antigen; CI: confidence interval.

\section{Supplementary Material}

Supplementary figure S1.

http://www.jcancer.org/v09p1642s1.pdf

\section{Acknowledgements}

This work was supported by grants from the the National Natural Science Foundation of China (No. 81672989); Jiangsu Clinical medicine Science and Technology Special Fund (BL2014091); Jiangsu Provincial Commission of Health and Family Planning Youth Research Project (Q201601).

\section{Funding sources for the study}

The National Natural Science Foundation of China (No. 81672989); Jiangsu Provincial Commission of Health and Family Planning Youth Research Project (Q201601); The National Natural Science Foundation of China (No. 81702685); The National Natural Science Foundation of China (No. 81702693); The National Natural Science Foundation of China (No. 81602381) and National Science Foundation of Jiangsu Province (BK 20151019). 


\section{Competing Interests}

The authors have declared that no competing interest exists.

\section{References}

1. Torre LA, Bray F, Siegel RL, Ferlay J, Lortet-Tieulent J, Jemal A. Global cancer statistics. 2012. CA: a cancer journal for clinicians. 2015; 65:87-108.

2. Cao SM SM, Qian $\mathrm{CN}$. the prevalence and prevention of nasopharyngeal carcinoma in china. Chin J Cancer. 2011.

3. Lee AW, Sze WM, Au JS, Leung SF, Leung TW, Chua DT, et al. Treatment results for nasopharyngeal carcinoma in the modern era: the Hong Kong experience. International journal of radiation oncology, biology, physics. 2005; 61:1107-16.

4. Wang HY, Chang YL, To KF, Hwang JS, Mai HQ, Feng YF, et al. A new prognostic histopathologic classification of nasopharyngeal carcinoma. Chin J Cancer. 2016; 35:41.

5. Al-Sarraf M, LeBlanc M, Giri PG, Fu KK, Cooper J, Vuong T, et al. Chemoradiotherapy versus radiotherapy in patients with advanced nasopharyngeal cancer: phase III randomized Intergroup study 0099. Journal of clinical oncology: official journal of the American Society of Clinical Oncology. 1998; 16:1310-17.

6. Lin JC, Jan JS, Hsu CY, Liang WM, Jiang RS, Wang WY. Phase III study of concurrent chemoradiotherapy versus radiotherapy alone for advanced nasopharyngeal carcinoma: positive effect on overall and progression-free survival. Journal of clinical oncology: official journal of the American Society of Clinical Oncology. 2000; 21:631-37.

7. Kwong DL, Sham JS, Au GK, Chua DT, Kwong PW, Cheng AC, et al. Concurrent and adjuvant chemotherapy for nasopharyngeal carcinoma: a factorial study. Journal of clinical oncology: official journal of the American Society of Clinical Oncology. 2004; 22:2643-53.

8. Chan AT, Teo PM, Ngan RK, Leung TW, Lau WH, Zee B, et al. Concurrent chemotherapy-radiotherapy compared with radiotherapy alone in locoregionally advanced nasopharyngeal carcinoma: progression-free survival analysis of a phase III randomized trial. Journal of clinical oncology: official journal of the American Society of Clinical Oncology. 2002; 20:2038-44

9. Wee J, Tan EH, Tai BC, Wong HB, Leong SS, Tan T, et al. Randomized trial of radiotherapy versus concurrent chemoradiotherapy followed by adjuvant chemotherapy in patients with American Joint Committee on Cancer/International Union against cancer stage III and IV nasopharyngeal cancer of the endemic variety. Journal of clinical oncology: official journal of the American Society of Clinical Oncology. 2005; 23:6730-38

10. Lee AW, Tung SY, Ngan RK, Chappell R, Chua DT, Lu TX, et al. Factors contributing to the efficacy of concurrent-adjuvant chemotherapy for locoregionally advanced nasopharyngeal carcinoma: combined analyses of NPC-9901 and NPC-9902 Trials. European journal of cancer (Oxford, England: 1990). 2011; 47:656-66.

11. al-Sarraf M, Pajak TF, Cooper JS, Mohiuddin M, Herskovic A, Ager PJ: Chemo-radiotherapy in patients with locally advanced nasopharyngeal carcinoma: a radiation therapy oncology group study. Journal of clinical oncology: official journal of the American Society of Clinical Oncology. 1990; 8:1342-51.

12. Lin JC, Liang WM, Jan JS, Jiang RS, Lin AC. Another way to estimate outcome of advanced nasopharyngeal carcinoma--is concurrent chemoradiotherapy adequate? International journal of radiation oncology, biology, physics. 2004; 60:156-64.

13. Ma BB, Poon TC, To KF, Zee B, Mo FK, Chan CM, et al. Prognostic significance of tumor angiogenesis, Ki 67, p53 oncoprotein, epidermal growth factor receptor and HER2 receptor protein expression in undifferentiated nasopharyngeal carcinoma--a prospective study. Head \& neck, 2003; 25:864-72.

14. Bonner JA, Harari PM, Giralt J, Azarnia N, Shin DM, Cohen RB, et al. Radiotherapy plus cetuximab for squamous-cell carcinoma of the head and neck. The New England journal of medicine. 2006; 354:567-78.

15. Sung FL, Poon TC, Hui EP, Ma BB, Liong E, To KF, et al. Antitumor effect and enhancement of cytotoxic drug activity by cetuximab in nasopharyngeal carcinoma cells. In vivo (Athens, Greece). 2005; 19:237-45.

16. Ma BB, Kam MK, Leung SF, Hui EP, King AD, Chan SL, et al. A phase II study of concurrent cetuximab-cisplatin and intensity-modulated radiotherapy in locoregionally advanced nasopharyngeal carcinoma. Annals of oncology: official journal of the European Society for Medical Oncology. 2012; 23:1287-92

17. He X, Xu J, Guo W, Jiang X, Wang X, Zong D. Cetuximab in combination with chemoradiation after induction chemotherapy of locoregionally advanced nasopharyngeal carcinoma: preliminary results. Future oncology. 2013; 9:1459-67.

18. Cox JD, Stetz J, Pajak TF. Toxicity criteria of the Radiation Therapy Oncology Group (RTOG) and the European Organization for Research and Treatment of Cancer (EORTC). International journal of radiation oncology, biology, physics. $1995 ; 31: 1341-46$
19. D'Agostino RB, Jr. Propensity score methods for bias reduction in the comparison of a treatment to a non-randomized control group. Statistics in medicine. 1998; 17: 2265-81.

20. Kang $\mathrm{H}$, Kiess A, Chung $\mathrm{CH}$. Emerging biomarkers in head and neck cancer in the era of genomics. Nature reviews Clinical oncology. 2015; 12:11-26.

21. Huang SM, Bock JM, Harari PM. Epidermal growth factor receptor blockade with C225 modulates proliferation, apoptosis, and radiosensitivity in squamous cell carcinomas of the head and neck. Cancer research. 1999; 59:1935-40.

22. Frampton JE. Spotlight on cetuximab in squamous cell carcinoma of the head and neck. Bio Drugs: clinical immunotherapeutics, biopharmaceuticals and gene therapy. 2011; 25:129-33.

23. Frampton JE. Cetuximab: a review of its use in squamous cell carcinoma of the head and neck. Drugs. 2010; 70:1987-2010.

24. Bonner JA, Harari PM, Giralt J, Cohen RB, Jones CU, Sur RK, et al. Radiotherapy plus cetuximab for locoregionally advanced head and neck cancer: 5-year survival data from a phase 3 randomised trial, and relation between cetuximab-induced rash and survival. The Lancet Oncology. 2010; 11:21-28.

25. Ang KK, Zhang Q, Rosenthal DI, Nguyen-Tan PF, Sherman EJ, Weber RS, et al: Randomized phase III trial of concurrent accelerated radiation plus cisplatin with or without cetuximab for stage III to IV head and neck carcinoma: RTOG 0522. Journal of clinical oncology: official journal of the American Society of Clinical Oncology. 2014; 32:2940-50.

26. Araki D, Redman MW, Martins R, Eaton K, Baik C, Chow L, et al. Concurrent cetuximab and postoperative radiation in resected high-risk squamous cell carcinomas of the head and neck: A single-institution experience. Head \& neck. 2016; 38:1318-23.

27. Zhang X, Du L, Zhao F, Wang Q, Yang S, Ma L. A Phase II Clinical Trial of Concurrent Helical Tomotherapy plus Cetuximab Followed by Adjuvant Chemotherapy with Cisplatin and Docetaxel for Locally Advanced Nasopharyngeal Carcinoma. International journal of biological sciences. 2016; 12:446-53.

28. $\mathrm{Xu} \mathrm{T}, \mathrm{Ou} \mathrm{X}$, Shen $\mathrm{C}, \mathrm{Hu}$ C. Cetuximab in combination with chemoradiotherapy in the treatment of recurrent and/or metastatic nasopharyngeal carcinoma. Anti-cancer drugs. 2016; 27:66-70.

29. Xu T, Liu Y, Dou S, Li F, Guan X, Zhu G, et al. Weekly cetuximab concurrent with IMRT aggravated radiation-induced oral mucositis in locally advanced nasopharyngeal carcinoma: Results of a randomized phase II study. Oral oncology. 2015; 51:875-79.

30. Wu X, Huang J, Liu L, Li H, Li P, Zhang J, et al. Cetuximab concurrent with IMRT versus cisplatin concurrent with IMRT in locally advanced nasopharyngeal carcinoma: A retrospective matched case-control study. Medicine. 2016; 95:e4926.

31. Feng HX, Guo SP, Li GR, Zhong WH, Chen L, Huang LR, et al. Toxicity of concurrent chemoradiotherapy with cetuximab for locoregionally advanced nasopharyngeal carcinoma. Medical oncology. 2014; 31:170.

32. Bar-Ad V, Zhang OE, Harari PM, Axelrod R, Rosenthal DI, Trotti A, et al. Correlation Between the Severity of Cetuximab-Induced Skin Rash and Clinical Outcome for Head and Neck Cancer Patients: The RTOG Experience. International journal of radiation oncology, biology, physics. 2016; 95:1346-54.

33. Zeng L, Tian YM, Sun XM, Chen CY, Han F, Xiao WW, et al. Late toxicities after intensity-modulated radiotherapy for nasopharyngeal carcinoma: patient and treatment-related risk factors. British journal of cancer. 2014; 110:49-54 